\title{
Path Loss Model Considering Doppler Shift for High Speed Railroad Communication
}

\author{
Junyeong Bok and Heung-Gyoon Ryu \\ Department of Electronic Engineering, Chungbuk National University, Korea \\ bjy84@nate.com, ecomm@cbu.ac.kr
}

\begin{abstract}
In this paper, we propose the tuned free-space path loss modelling in high speed railroad considering Doppler effect. We use tuned free space path loss model which is utilized for measurement results at high speed railroad. The environment of high speed rail is mostly at viaduct and flatland over than 50 percent. The purpose of this paper is analyzing Doppler shift effect at viaduct and plain by using modified path loss model. Simulation results show that proposed path loss model considering estimated Doppler shift coincides with the free space loss model.
\end{abstract}

Keywords — Path loss, high speed railroad, Doppler shift, free space, propagation.

\section{INTRODUCTION}

The demand for wireless communication consistently increases in high-speed mobile environment due to the development of high-speed railroad. It is important to provide better link quality be caused by the train's speed up and data rate increase. Beam-forming technique can be used widely in order to provide link quality as enhancing the strength of the transmitted signal or as reducing interference signal. But, getting the channel state information at base station is difficult, when high speed railroad communication uses beam-forming technique. Therefore, path loss model is very important in order to design consider diverse characteristic in high-speed train communication.

There are many models for analysing the path loss model nevertheless any path loss model can not apply to comprehensive. There have been studies on path loss between transmitter and receiver at urban and suburban environment [1-2]. However, there are few researches about path loss at viaduct and plain. But, path loss model is very important in the design of high-speed rail communication system, it is necessary to analyse accurately the Doppler shift effect.

There are a various path loss modelling methods such as using Ray tracing [3], concrete morphological and using building data [4], and previous model adding average residuals [5]. But, reference [5] has path loss exponent and actually path loss exponent is affected by obstacles between transmitter and receiver [6]. The Doppler effect is generated largely at high speed railroad. Therefore, there are needs to research about path loss modelling considering the Doppler effect.

In this paper, we propose tuned free path loss model considering Doppler shift at viaduct and plain in high-speed train environment. We confirm that there is almost no error between estimated model and tuned free space path loss model.

\section{Measurement EnVironment}

Path loss information is measured at train for path loss modelling of high speed railroad. Hence, maximum velocity of train is $340 \mathrm{~km} / \mathrm{h}$. The flat land and viaduct have similar transfer characteristics because of low fluctuation. Measurement distances at viaduct and at flatland are $3 \mathrm{~km}$ and $1.793 \mathrm{~km}$. Test frequency of railway network is downlink frequency with $200 \mathrm{KHz}$ bandwidth from 930.2 MHz to 933.4 $\mathrm{MHz}$ range. Transmitting antenna on the line has $17 \mathrm{dBi}$ gain of beam which is the cross polarized $60^{\circ}$ horizontal and $6.8^{\circ}$ vertical beam width. Hence, antenna transmit power is $43 \mathrm{dBm}$, and omni-direction receiver antenna is located $3.5 \mathrm{~m}$ high above the train.

\section{Tuned Free-Space Path Loss Model}

Free-space path loss is calculated based on the following

$$
L_{0}(d B)=32.45+20 \times \log _{10}(d)+20 \times \log _{10}(f)
$$

where $d$ is distance $(\mathrm{km})$ between transmitter and receiver, $f$ is carrier frequency $(\mathrm{MHz})$. Path loss through measured data is calculated using the following equation

$$
L_{m}=P_{t}+G_{b}-l_{b}-l_{d}-P_{r}
$$

where $P_{r}$ is $43 \mathrm{dBm}$ as receiver power and $P_{t}$ is $43 \mathrm{dBm}$ as transmission power. $G_{b}$ is $17 d B i$ as transmitter antenna gain, $l_{b}$ is a feeder loss at base band, $l_{d}$ is as power divider. The difference between free-space and the measured path loss is given by

$$
\Delta L=L_{m}-L_{0}
$$

Path loss is linearly proportional in log domain. Distance between the receiver and base station is only changed at each time. Therefore, tuned equation is defined as

$$
\Delta L=K_{1} \log _{10} d+K_{2}
$$

where $K_{1}$ is parameter about distance and $K_{2}$ is constant. Two parameters can be obtained by the Least-square criterion.

$K_{1}$ and $K_{2}$ are applied median value in [7]. The finally value is obtained as $K_{1}=6.26, K_{2}=9.855$ and 
$K_{1}=14.19, K_{2}=12.72$ at viaduct and plain. Therefore, tuned free space path loss model at viaduct and plain can be expressed as

$$
\begin{gathered}
L=\Delta L+L_{0} \\
L_{v i a}=42.305+26.26 \times \log _{10}(d)+20 \times \log _{10}(f) \\
L_{p l a}=46.17+34.19 \times \log _{10}(d)+20 \times \log _{10}(f)
\end{gathered}
$$

\section{IV.ESTIMATION MODEL CONSIDERING DOPPLER SHIFT}

Variation the reception frequency is generated by Doppler effect due to movement of transmitter and receiver.

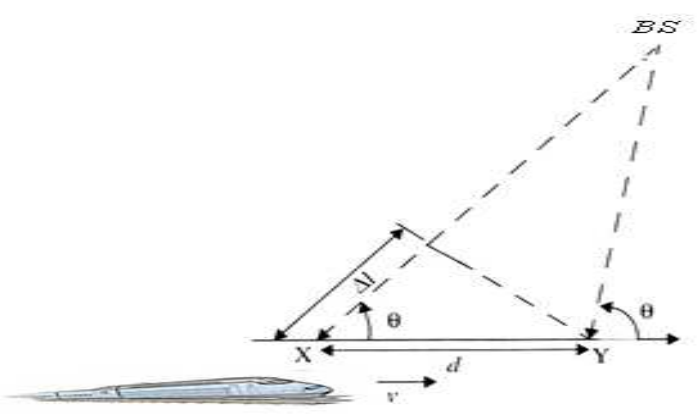

Figure 1. Doppler shift effct.

Doppler shift effect is related to the velocity of mobile device and angle between reception radio wave and the moving direction in figure 1 . If variation of received frequency occurs, it is possible as variation factor of carrier frequency recognize as a loss of received signal without compensation for Doppler effect.

When train with $\mathrm{v}$ velocity moves from $\mathrm{X}$ to $\mathrm{Y}$, difference of paths $\Delta l$ is defined as

$$
\Delta l=d \cos \theta=v \Delta t \cos \theta
$$

where $\theta$ is incoming angle between base station and trans. Phase change $\Delta \varphi$ is given by

$$
\Delta \varphi=\frac{2 \pi \Delta l}{\lambda}=\frac{2 \pi \Delta t}{\lambda} \cos \theta
$$

Therefore, frequency variation by Doppler effect can be expressed as

$$
f_{d}=\frac{1}{2 \pi} \frac{\Delta \phi}{\Delta t}=\frac{v}{\lambda} \cos \theta=v \frac{f_{c}}{c} \cos \theta
$$

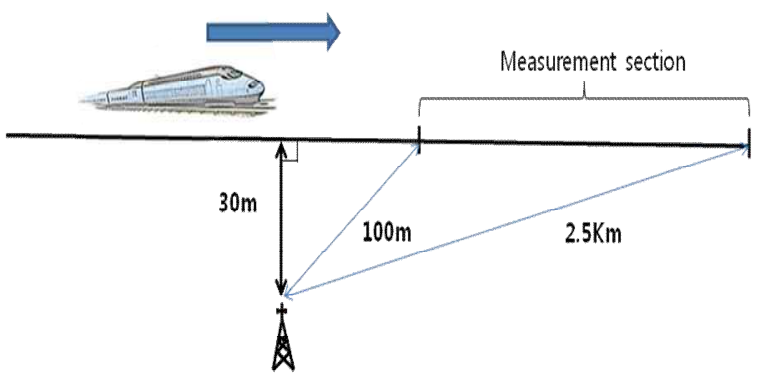

Figure 2. Simulation condition.

Fig. 2 shows simulation environment for estimation model considering Doppler shift. In this paper, we assume distance between base station and the rail is $30 \mathrm{~m}$. Also, we measure at $100 \mathrm{~m}$ unit from $100 \mathrm{~m}$ to $2.5 \mathrm{~km}$ about distance from base station to train in order to improve reliability of results. Therefore, we estimate novel estimation model with minimum error between estimated novel path loss model values and tuned path loss model values. Carrier frequency $f_{c}$ is $930 \mathrm{MHz}$ and maximum velocity of trains is $340 \mathrm{Km} / \mathrm{h}$ $(=94.4 \mathrm{~m} / \mathrm{s})$.

High speed train's channel model is changed by location of transceiver, the height of the viaduct, and antenna location. We can estimate free space path loss model of viaduct and plain considering these points. But there are the difference between the free-space path loss and the measurement values. We confirm that these two models have difference at measurement range of simulation environment in figure 3 . Therefore, tuned free space model is need.

Tuned path loss model represents the sum form of median values of measurement values at existing free space path loss model. Residuals have $K_{1} \times \log _{10}(d)+K_{2}$ form, $K_{1}$ and $K_{2}$ are obtained through measurement results.

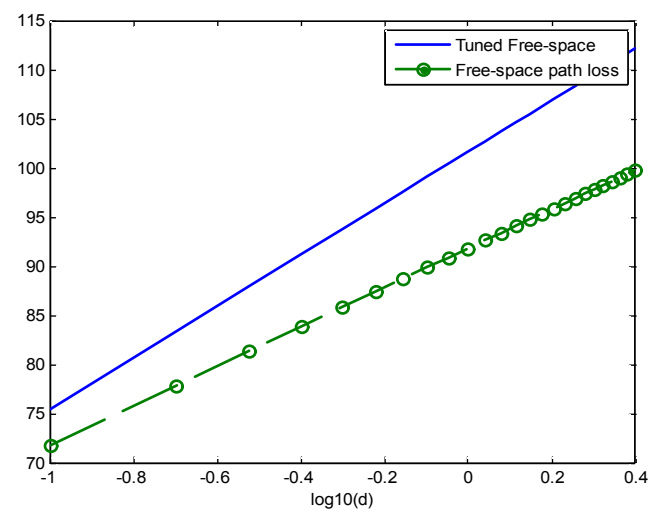

Figure 3. Tuned Free space model and path loss model at viaduct.

This paper, novel tuned path loss model considering Doppler shift effect can be expressed as

$$
\begin{aligned}
L= & K_{1} \times \log _{10}\left(f_{d}\right)+K_{2} \times \log _{10}(d)+K_{3} \\
& \underbrace{+32.45+20 \times \log _{10}(d)+20 \times \log _{10}(f)}_{\text {Free-space pathloss model }}
\end{aligned}
$$

where frequency change occurs due to Doppler shift.

In order to determine parameters $K_{1}, K_{2}$ and $K_{3}$, we select optimum values with the minimum error between tuned path loss model and measured data, where step size is 0.1 . The final parameters at viaduct and at plain represents as

$$
\begin{gathered}
K_{1}=6.2, K_{2}=12.5, K_{3}=4.0 \text { in Viaduct } \\
K_{1}=5.0, K_{2}=19.2, K_{3}=9.0 \text { in Plain }
\end{gathered}
$$

The estimated path loss model considering Doppler shift can be expressed as

$$
\begin{aligned}
& L_{\text {via }}=6.2 \times \log _{10}\left(f_{d}\right)+32.5 \times \log _{10}(d)+20 \times \log _{10}(f) \\
& L_{\text {pla }}=5.0 \times \log _{10}\left(f_{d}\right)+39.2 \times \log _{10}(d)+20 \times \log _{10}(f)+41.45
\end{aligned}
$$



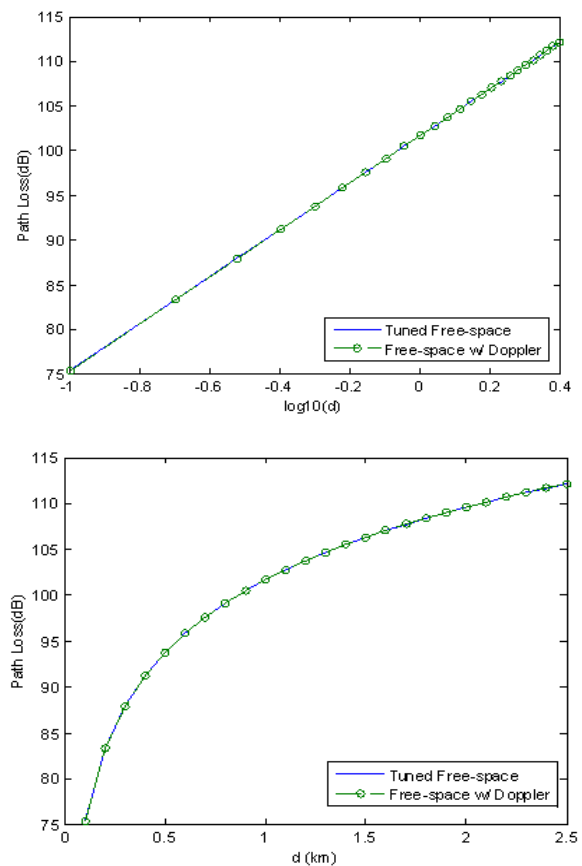

Figure 4. Comparison of the path loss of tuned Free-space model and Freespace model with Doppler shift in Viaduct.
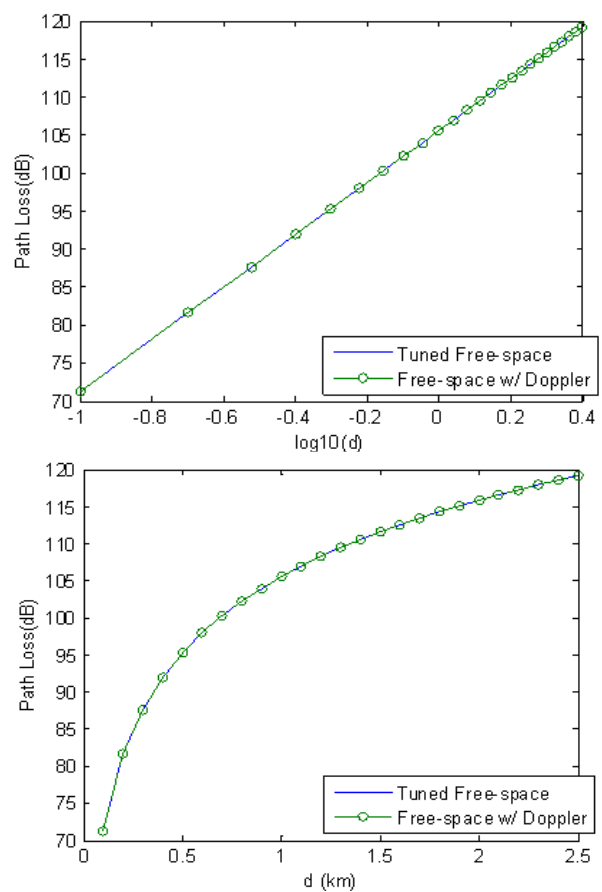

Figure 5. Comparison of the path loss of tuned Free-space model and Freespace model with Doppler shift in Plain.

Fig. 4 and Fig. 5 show difference of reference [7] and proposed path loss consider Doppler shift at viaduct and plain respectively. Simulation results shows that the proposed path loss estimation model is generally accurate compare to previous schemes.

\section{CONCLUSION}

In this paper, we investigate the propagation model considering Doppler effect by using measured data of high speed railroad at viaduct and plain in the free space. We confirm that the estimated model is almost similar the tuned free space path loss model. However, the influence of path loss model due to Doppler shift is very small actually. In the future work, we consider more variety of environments for accurate estimation of path loss model in high speed railroad.

\section{ACKNOWLEDGMENT}

"This research was supported by Basic Science Research Program through the National Research Foundation of Korea(NRF) funded by the Ministry of Education, Science and Technology (No. 2012017339) and (No. 2013R1A2A2A0 1005849)"

\section{REFERENCES}

[1] T.H. Keith, W.H. Jack, H.S. David, and W.B. Daniel, "Path-Los Characteristics of Urban Wireless Channels," IEEE Trans. Antennas Propag., vol. 58, no.1, pp. 171-177, Jan. 2010.

[2] V. Erceg, L.J. Greenstein, S.Y. Tjandra, S.R. Parkoff, A. Gupta, B Kulic, A.A. Julius, and R. Bianchi, "An Empirically Based Path Los Model for Wireless Channels in Suburban Environments," IEEE J. Select. Areas in Commun., vol. 17, issue. 7, pp. 1205-1211, Jul. 1999.

[3] A. Amornthipparat, H. Shirai, K. Yonezawa, T. Inoue, and Y Nakamura, "Estimation of High Frequency NLOS Path loss in StreetCell Environment," ICCE 2008. Second International Conf., pp. 336340, Jun. 2008.

[4] P. Schneider, F. Lambrecht, and A. BaierP, "Enhancement of the Okumura Hata propagation model using detailed morphological and building data," Personal, Indoor and Mobile Radio Communications, 7th IEEE International Symposium, vol. 1, pp. 34-38, Oct. 1996.

[5] J. Turkka and M. Renfors, "Path Loss Measurements for a Non-Line-of Sight Mobile-to-Mobile Environment," ITS Telecommunications, 8th International Conf., pp. 274-278, Oct. 2008.

[6] M. C. Walden, F.J. Rowsell, "Urban Propagation Measurements and Statistical Path Loss Model at 3.5GHz," IEEE Antennas and Propagation Society International Symposium, vol. 1A, pp. 363-366, Jul. 2005.

[7] W. Hong, Z. Zhangdui, G. Ke, and A. Bo, "Path Loss Models in Viaduct and Plain Scenarios of the High-speed Railway," CHINACOM 2010 5th International ICST Conference, pp.1-5, Aug. 2010.

[8] J.G. Proakis and M. Salehi "Essentials of Communication Systems Engineering" N.J.: Pearson Prentice Hall, 2005.

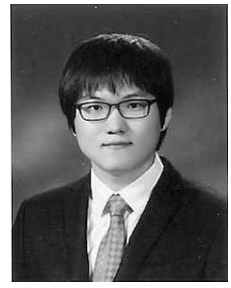

antenna system.

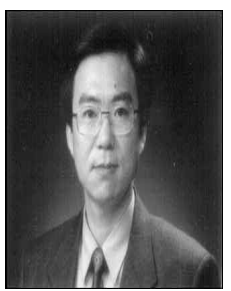

Junyeong Bok was born in Chungnam, Republic of Korea in 1985. He received the B.S. and M.S degrees in the department of electronic engineering from Chungbuk National University in 2010 and 2012. Now he is currently working toward Ph.D. degree at the department of Electronic Engineering, Chungbuk National University, Republic of Korea. His research interests include digital communication system, OFDM communication system and digital retrodirective array

Heung-Gyoon Ryu (M'88) was born in Seoul, Republic of Korea in 1959. He received the B.S. and M.S. and Ph.D. degrees in electronic engineering from Seoul National University in 1982, 1984 and 1989. Since 1988, he has been with Chungbuk National University, Korea, where he is currently Professor of 
Department of Electrical, Electronic and Computer Engineering in Chungbuk National University. And he worked as Chief of RICIC (research institute of computer, information communication center) in Chungbuk National University from March 2002 to Feb 2004. His main research interests are digital communication systems, communication circuit design, spread spectrum system and communication signal processing. Since 1999, he has worked as reviewer of the IEEE transaction paper. He was a winner of '2002 ACADEMY AWARD' from the Korea Electromagnetic Engineering Society, Korea. He received the "BEST PAPER AWARD" at the 4th International Conference on Wireless Mobile Communications (ICWMC 2008) Athens, Greece, July 27-Aug.1, 2008. Also, He received the "BEST PAPER AWARD" at the International Conference on Advances in Satellite and Space Communications (SPACOMM 2009), Colmar France, July 20-25, 2009. 